DOI: 10.12957/demetra.2018.33495

\title{
Atitudes alimentares e imagem corporal das mães de pacientes com transtornos alimentares
}

\section{Eating behavior and body image of mothers of patients with eating disorders}

Caroline Lara Garcia

Raphaela Meneghetti Miguel

Rosane Pilot Pessa ${ }^{2}$

Marina Garcia Manochio-Pina ${ }^{3}$

1 Universidade de Franca, Curso de Graduação em Nutrição. Franca, SP, Brasil.

${ }^{2}$ Universidade de São Paulo, Escola de Enfermagem de Ribeirão Preto, Departamento de Enfermagem Materno-Infantil e Saúde Pública. Ribeirão Preto, SP, Brasil.

${ }^{3}$ Universidade de Franca, Programa de Mestrado e Doutorado em Promoção de Saúde. Franca, SP, Brasil.

Correspondência / Correspondence Marina Garcia Manochio-Pina E-mail:mamanochio@yahoo.com.br

\section{Resumo}

Introdução: Transtornos alimentares são definidos como doenças psicossomáticas resultantes de uma relação conflituosa do indivíduo com a comida. Objetivo: Investigar o comportamento alimentar e avaliar a percepção e a satisfação com a imagem corporal de mães de pacientes com transtornos alimentares. Metodologia: Trata-se de pesquisa quantitativa, realizada em serviço especializado no interior de São Paulo. Os métodos utilizados foram Recordatório de 24 horas, o teste "Como está sua Alimentação", o EAT- 26 e a Escala de Figuras de Silhuetas. Participaram do estudo 13 mães, das 18 pacientes atendidas pelo programa. Resultados: O EAT-26 foi negativo, com 17,23 pontos, o teste "Como está sua Alimentação" mostrou a média de 38,85 pontos. Em relação à imagem corporal, notou-se insatisfação por parte das mães. Discussão: As mães apresentam insatisfação com a imagem corporal, apesar de não apresentarem comportamentos alimentares semelhantes aos hábitos de pacientes com o transtorno. Conclusão: Poucos estudos foram realizados avaliando o comportamento alimentar de mães de pacientes com transtornos alimentares, portanto é necessário realizar mais pesquisas envolvendo esse aspecto. É necessária a participação da família no tratamento para que este seja bem-sucedido.

Palavras-chave: Transtorno Alimentar. Atitudes Alimentares. Imagem Corporal. Mães. 


\section{Abstract}

Introduction: Eating disorders are defined as psychosomatic illnesses resulting from a conflicting relationship of the individual with the food. Objective: the study aimed to investigate the eating behavior and to evaluate the perception and satisfaction with the body image of mothers of patients with eating disorders. Methodology: It is a quantitative research carried out in a specialized service in the interior of São Paulo. The methods used were a 24-hour Reminder, the "How is Your Food" test, the EAT-26 and the Silhouetted Figures Scale. Thirteen mothers participated in study, out of the 18 patients attended by the program. Results: the EAT-26 was negative, with 17.23 points, the How-to-eat test showed an average of 38.85 points. Discussion: Regarding body image, mothers' dissatisfaction was noted. The study concludes that mothers are dissatisfied with body image, although they do not present eating behaviors similar to the habits of patients with the disorder. Conclusion: Few studies have been conducted evaluating the eating behavior of patient's mother with eating disorders, so it is necessary to carry out more research involving this aspect. Family participation in treatment is necessary to achieve success.

Keywords: Eating disorder. Food Attitudes. Body Image. Mother.

\section{Introdução}

Transtornos alimentares (TA) são definidos como doenças psicossomáticas resultantes de uma relação conflituosa do indivíduo com a comida. Os tipos de TA mais conhecidos são a anorexia nervosa (AN) e a bulimia nervosa (BN), ambos apresentando como sintoma primário a preocupação com o peso e a forma corporal. Os primeiros sinais dos TA aparecem, na maioria das vezes, na infância e na adolescência, sendo mais comum em mulheres e considerados de difícil tratamento devido a sua etiologia multifatorial. A formação de uma equipe multiprofissional, composta por médico, nutricionista e psicólogo, é essencial para alcançar resultados satisfatórios no tratamento. ${ }^{1-4}$

Os fatores que levam ao aparecimento e/ou manutenção dos quadros de TA são uma questão frequentemente invocada tanto por profissionais da área da saúde ${ }^{5}$ como pelos familiares, que buscam incessantemente respostas para a genealogia dos sintomas. ${ }^{6}$

Logo após o nascimento, fatores culturais e psicossociais podem influenciar as experiências alimentares das crianças, proporcionando a aprendizagem inicial para a sensação de fome, saciedade e para a percepção dos sabores. As estratégias que os pais utilizam para introduzir a 
alimentação da criança e ensiná-la a comer alimentos específicos podem apresentar estímulos tanto adequados quanto inadequados na obtenção das preferências alimentares da criança e no autocontrole da ingestão alimentar. ${ }^{7}$

A partir das primeiras descrições dos TA, algumas características do funcionamento familiar vêm sendo investigadas, e atualmente estão recebendo atenção especial e adquirindo relevância do ponto de vista etiológico. Vários estudos buscam relacionar o funcionamento familiar do paciente como fator desencadeador ou perpetuador do quadro. Certas características da dinâmica familiar do paciente, como baixa capacidade de expressão emocional, baixo nível de coesão e experiência de conflitos mais intensos do que nas famílias empregadas como controles, são frequentemente mencionadas pela literatura. A relação mãe-filha é estudada, pois é caracterizada por predomínio de uma relação fusional e simbiótica e pela presença de um apego inseguro e nível de conflito elevado. ${ }^{6}$ Para Kaës, ${ }^{7}$ a transmissão transgeracional, que é a transmissão da doença de geração para geração, ou seja, uma transmissão familiar que implica falta do limite e do espaço subjetivo, permanecendo apenas a exigência do narcisismo. São elementos transmitidos através dos sujeitos e não entre eles, perpetuando segredos e lutos que dificultam a transformação e a simbolização. ${ }^{8}$

Outros estudos apontam para a transmissão psíquica que é realizada entre as gerações, sendo os TA exemplos desta transmissão. Pereira, Lock \& Oggins ${ }^{9}$ dizem que é fundamental que a família também se desempenhe na formação de uma aliança com o tratamento de suas filhas com AN, uma vez que isso pode contribuir para o desfecho favorável do tratamento. ${ }^{10}$ Geralmente as mães estão muito presentes desde a infância das crianças e podem ser grandes aliadas no tratamento de suas filhas com TA. Através do conhecimento dos hábitos alimentares, avaliações nutricionais e questionários com as mães das pacientes, podem-se encontrar resultados que mostrem a real influencia principalmente das mães, no desenvolvimento dos TA das filhas, buscando assim outras formas de tratamento para as mesmas, envolvendo sobretudo a figura materna.

O objetivo do estudo foi investigar o comportamento alimentar de mães de pacientes com TA e avaliar sua percepção e satisfação com a imagem corporal.

\section{Método}

Trata-se de pesquisa transversal com delineamento quantitativo realizada com mães de pacientes com TA em um serviço especializado no interior do estado de São Paulo.

Participaram da pesquisa 13 mães das 18 pacientes que estavam em atendimento no período da coleta de dados, já que uma mãe não aceitou participar da pesquisa, duas já haviam falecido e duas não compareceram. 
A coleta de dados aconteceu em salas individuais e reservadas, sendo a identidade das mães mantida em sigilo. Todos os testes foram respondidos pelas próprias mães e a coleta durou cerca de 30 minutos para cada participante.

Foi avaliado o estado nutricional através do Índice de Massa Corporal (IMC), para cujo cálculo são necessários apenas o peso e estatura das mães. Esses procedimentos, de pesar e aferir a estatura das mães, seguiram as orientações contidas no Manual do Sistema de Vigilância do Ministério da Saúde. ${ }^{11}$

Foram coletados dados sobre a ingestão alimentar através do recordatório de 24 horas, e assim foi possível verificar os grupos da pirâmide alimentar que elas consumiam. ${ }^{12}$

O teste desenvolvido pelo Ministério da Saúde, "Como está sua alimentação", consiste em perguntas sobre a alimentação do participante onde cada alternativa de resposta possui uma pontuação. Ao final do teste são somados os pontos; se o resultado for de até 28 pontos, o participante deverá tornar sua alimentação e seus hábitos de vida mais saudáveis; se o resultado for de 29 a 42 pontos, o participante deverá ficar atento com sua alimentação e outros hábitos como atividade física e consumo de líquidos; se o resultado for maior que 43 pontos, o participante estará no caminho certo para o modo de vida saudável. ${ }^{13}$

Foi utilizado também o Eating Attitudes Test (EAT-26), que indica a presença de padrões alimentares inadequados e fornece um índice de gravidade de preocupações típicas de pacientes com TA, particularmente com intenção de emagrecer e medo de ganhar peso. ${ }^{14} \mathrm{O}$ EAT-26 é um dos questionários mais utilizados atualmente na área de TA, por ser um teste psicométrico autoaplicável de rastreamento, para identificar sinais e sintomas desses quadros de maneira mais rápida e fácil. Favorece, assim, a precocidade do diagnóstico e tratamento. Foi construído por Garner \& Garfinkel ${ }^{15}$ e validado para a população brasileira. Contém 26 questões divididas em três escalas ou fatores obtidas através de análise fatorial: (i) Escala da Dieta (D) - itens n ${ }^{\circ}$ 1, 6, 7, 10, 11, 12, 14, 16, 17, 22, 23, 24, 25: reflete uma recusa patológica a comidas de alto valor calórico e preocupação intensa com a forma física; (ii) Escala de Bulimia e Preocupação com os Alimentos (B) - itens $n^{\circ} 3$, 4, 9, 18, 21, 26: refere-se a episódios de ingestão compulsiva dos alimentos, seguidos de vômitos e outros comportamentos para evitar o ganho de peso; (iii) Escala do Controle Oral (CO) - itens $\mathrm{n}^{\circ} 2,5,8,13,15,19,20$ : demonstram o autocontrole em relação aos alimentos e reconhece forças sociais no ambiente que estimulam a ingestão alimentar.

Cada questão, dividida em três escalas do tipo Likert, apresenta seis opções de resposta, conferindo-se pontos de zero a três, dependendo da escolha (sempre $=$ três pontos, muitas vezes $=$ dois pontos, às vezes $=$ um ponto, poucas vezes $=$ zero ponto, quase nunca $=$ zero ponto e nunca $=$ zero ponto). A única questão que apresenta pontos em ordem invertida é a 25 . O resultado do teste é a soma de todos os pontos dados às questões, e acima de 21 pontos considera-se o teste positivo sugerindo fator de risco para o desenvolvimento de TA. ${ }^{16}$ 
A percepção e satisfação com a imagem corporal foram investigadas pela Escala de Figura de Silhuetas, instrumento desenvolvido para a população brasileira. Esse instrumento consiste na apresentação de uma determinada série de figuras que, geralmente, varia da figura mais magra até a mais gorda, onde o avaliado deve escolher a figura que representa seu corpo atual, ideal ou desejado. ${ }^{17}$ Essa escala é composta por 15 silhuetas para adultos de cada sexo, apresentadas em cartões plastificados individuais, com $12,5 \mathrm{~cm}$ de altura por $6,5 \mathrm{~cm}$ de largura. A figura branca centralizada sobre o fundo negro mede $10,5 \mathrm{~cm}$ de altura por $4,5 \mathrm{~cm}$ de largura, com variações progressivas na escala de medidas, da figura mais magra a mais larga, considerando-se inclusive a relação cintura-quadril. O IMC das silhuetas varia de $12,5 \mathrm{~kg} / \mathrm{m}^{2}$ a $47,5 \mathrm{~kg} / \mathrm{m}^{2}$, com intervalos constantes de $2,5 \mathrm{~kg} / \mathrm{m}^{2}{ }^{18}$

As variáveis numéricas estudadas no presente trabalho foram inicialmente descritas por parâmetros como média aritmética, desvio padrão e coeficiente de variação. Com a finalidade de definir a natureza paramétrica ou não-paramétrica das estatísticas de inferência, foram submetidas ao teste de normalidade de D’Agostino e Pearson que apontou para a normalidade das populações de origem. Sendo assim, as estatísticas utilizadas foram o teste $t$ de Student e o teste de KruskalWallis, que substituiu a ANOVA de Fisher para o caso de amostras parecidas. ${ }^{19}$

A Pesquisa foi aprovada pelo Comitê de Ética da Universidade, protocolo nํ47825415.3.3001.5440, e todos os participantes assinaram um termo de consentimento livre e esclarecido anteriormente à coleta de dados.

\section{Resultados}

As mães estudadas tinham entre 38 e 63 anos, com média de 49 anos. Todas moravam no interior do Estado de São Paulo, a maioria na região próxima à cidade de Ribeirão Preto (SP). Destas, $53,8 \%$ são casadas (n=7) e 46,2\% são solteiras $(n=6)$.

O “Teste Como Está sua Alimentação” mostrou uma média de 38,85 pontos ( $\mathrm{p}=0,1488)$. Com a análise do consumo dos macronutrientes, observou-se que a quantidade de proteína consumida foi de $19 \%$, sendo em média $0,9 \mathrm{~g} / \mathrm{kg}$ (limite inferior de referência $=10 \% ; \mathrm{p}=0,0005$ e limite superior de referência $=35 \% ; \mathrm{p}=<0,0001$ ). Ao comparar o consumo das porções alimentares das mães, com as porções da pirâmide alimentar, ilustrado na figura 1, pode-se perceber um consumo médio de 0,62 porções do grupo dos Feijões e Oleaginosa $(\mathrm{p}=0,054)$ e de 1,50 porções do grupo das Carnes e Ovos $(\mathrm{p}=0,072)$. Já no grupo do Leite, Queijo e Iogurte, a média de consumo foi de 0,92 porções $(\mathrm{p}=<0,0001)$. 


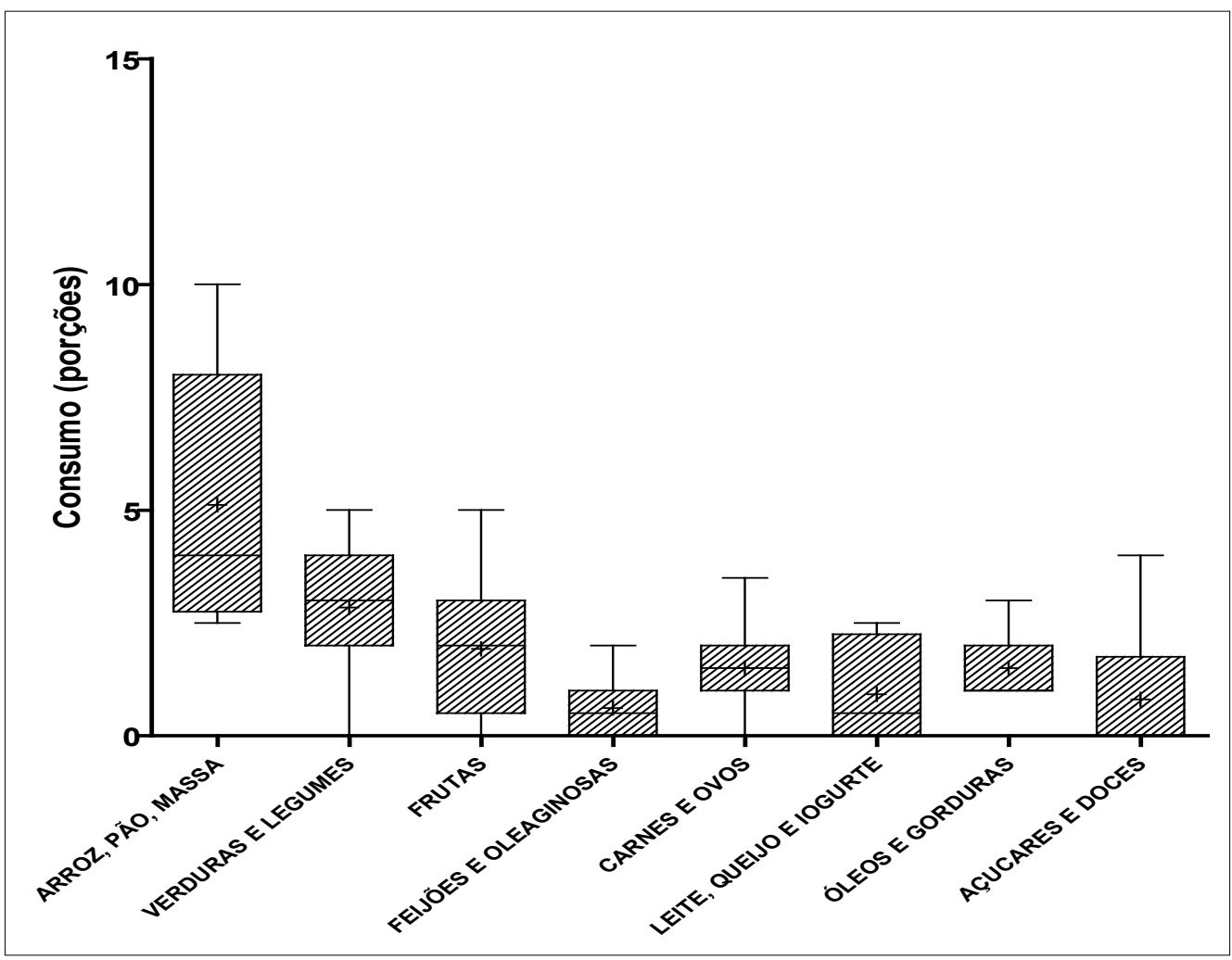

Figura 1. Consumo das Porções da Pirâmide Alimentar.

O consumo de carboidratos foi de 52,13\% (limite inferior de referência $=45 \% ; \mathrm{p}=0,040$ e limite superior de referência $=65 \% ; \mathrm{p}=0,002$ ), ao comparar com as porções da pirâmide, $o$ grupo do Arroz, Pães e Massas e o grupo das Verduras e Legumes, o consumo médio foi de respectivamente $5,12(\mathrm{p}=0,266)$ e 2,15 $(\mathrm{p}=0,726)$ porções consumidas. Já no grupo das Frutas, a média de consumo foi de 1,92 porções $(p=0,027)$. Ao avaliar o consumo do grupo dos Açúcares e Doces, pode-se notar um consumo médio de 0,81 porções $(\mathrm{p}=0,590)$.

A ingestão de lipídeos foi de 28,2\% (limite inferior de referência $=20 \% ; \mathrm{p}=0,0052$ e limite superior de referência $=35 \% ; \mathrm{p}=0,014$ ), ao comparar com as porções da pirâmide do grupo dos Óleos e Gorduras, o consumo foi em média de 1,5 porções $(p=0,016)$.

Em relação ao EAT-26, a média do grupo, projetada para a população, foi de 17,23 pontos $(\mathrm{p}=0,4299) ; 23,1 \%(\mathrm{n}=3)$ apresentaram resultados positivos para o teste. 
Em relação ao estado nutricional, 23,1\% (n=3) apresentaram Eutrofia, 23,1\% (n=3) apresentaram Sobrepeso, 38,4\% (n=5) com Obesidade Grau I e 15,4\% (n=2) Obesidade Grau II. Na Tabela 1, que mostra a percepção e satisfação da imagem corporal das mães, o IMC desejado, que representa a figura que as mães gostariam de ser, é significantemente menor que o IMC real, que representa o IMC aferido $(\mathrm{p}=0,016)$. Em relação ao IMC atual, que representa a figura de como elas se veem no momento, é significantemente maior que o IMC desejado, ou seja, elas superestimam o IMC atual em relação ao IMC desejado, mostrando assim uma insatisfação em relação ao IMC atual. O IMC real foi significantemente menor que o IMC atual, ou seja, elas superestimam o IMC real em relação ao atual $(p=0,016)$, mostrando uma inacurácia em relação a imagem corporal.

Tabela 1. Percepção e satisfação da imagem corporal das mães das pacientes do GRATA, segundo comparação entre o IMC $\left(\mathrm{kg} / \mathrm{m}^{2}\right)$ real e os correspondentes às figuras de silhuetas escolhidas no momento atual, em média (desvio-padrão). Ribeirão Preto, 2016.

\begin{tabular}{c|c|c|c|c|c|c}
\hline $\mathrm{IMC}_{\mathrm{A}}$ & $\mathrm{IMC}_{\mathrm{R}}$ & $\mathrm{IMC}_{\mathrm{D}}$ & $\begin{array}{c}\text { Diferença } \\
\mathrm{IMC}_{\mathrm{A}} \mathrm{e} \mathrm{IMC}_{\mathrm{R}}\end{array}$ & $\mathrm{p}$ & $\begin{array}{c}\text { Diferença } \\
\mathrm{IMC}_{\mathrm{A}} \mathrm{e} \mathrm{IMC}_{\mathrm{D}}\end{array}$ & $\mathrm{p}$ \\
\hline 34,23 & 29,08 & 24,81 & 5,5 & $0,016^{*}$ & 9,42 & $0,0018^{*}$ \\
$(10,38)$ & $(5,65)$ & $(6,88)$ & $\mathrm{IM}^{*}$ & & 9.5 \\
\hline
\end{tabular}

$\mathrm{IMC}_{\mathrm{A}=}$ Índice de Massa Corporal atual; $\mathrm{IMC}_{\mathrm{R}=}$ Índice de Massa Corporal real; $\mathrm{IMC}_{\mathrm{D}=}$ Índice de Massa Corporal desejado; *Diferença significativa.

\section{Discussão}

O binômio alimentar/ser alimentado (cuidar/ ser cuidado) é o primeiro grande organizador da vida psíquica do indivíduo. Considerando-se que o alimento é um fator que institui a relação mãe-bebê, veículo pulsional e agente de desejo, pode-se notar a importância do processo alimentar na vida emocional da criança. ${ }^{6}$ Nessa perspectiva, os hábitos de vida dos pais, os estilos parentais e a forma de como eles interagem com seus filhos são importantes para a formação dos hábitos alimentares infantis. A forma de como se dá a interação entre pais/cuidadores e filhos nos primeiros anos de vida repercute positiva ou negativamente na nutrição e no crescimento e desenvolvimento cognitivo e social da criança. Cuidadores que assumem o controle da alimentação e não reconhecem os sinais emitidos pela criança, pode fazer com que ela perca a sensibilidade à saciedade e a fome, contribuindo assim, para um ganho de peso elevado ou déficit nutricional. ${ }^{8,20}$

Pessoas com TA possuem relações características com suas mães. Estudo sobre a relação mãe e filha desde a gestação até os dois anos de idade mostrou que a alimentação é um forte elo entre as duas. As mães costumam reclamar de um sentimento de impotência e sofrimento; já as filhas 
são caracterizadas como vorazes, tendo assim, dificuldade em assimilar os cuidados oferecidos por sua mãe. Nesta complexa relação, o alimento é transformado em equivalente simbólico da função materna, sendo muitas vezes evitado ou até mesmo eliminado. ${ }^{21}$ Mães que falam muito sobre dietas e incentivam suas filhas a fazê-las podem ter uma grande influência no desenvolvimento do TA. ${ }^{22,23}$

Além da relação mãe-filha, também existem os fatores genéticos que podem estar associados. Em estudos realizados por Strober et al., ${ }^{24}$ com o objetivo de identificar a contribuição dos fatores genéticos para o desenvolvimento de TA, pode-se verificar que a incidência do transtorno em irmãs de pacientes com TA é de 6\%, aproximadamente, seis vezes maior que os valores mais altos encontrados em estudos populacionais. Outro estudo com 45 pares de gêmeos observou que a concordância para a manifestação do TA em dizigóticos foi de $5 \%$, enquanto que para monozigóticos foi de 56\%. Estes achados evidenciaram que os fatores genéticos têm papel importante na manifestação dos TA, o que confirma a grande importância da família no tratamento do TA. ${ }^{24}$

Destaca-se que as mães apresentam um ganho de peso importante durante o período de gestação e que, geralmente, esse peso adquirido dificilmente é perdido após o nascimento do bebê. Sabe-se que ganho de peso é fator de risco para o desenvolvimento de obesidade e TA; assim, é possível que a população de mães seja um grupo de risco para seu desenvolvimento. ${ }^{25}$

Ao analisar o teste "Como está sua Alimentação", pode-se concluir que essa população apresenta uma alimentação próxima ao considerado saudável, já que os resultados indicam pontuação maior que 29 pontos, que é seu limite inferior. Porém não houve diferença significativa em seu limite superior, o limite de alerta, que é de 42 pontos, o que nos leva a concluir que, embora esta população esteja dentro do intervalo de alerta, sua média populacional está na região limítrofe para uma boa alimentação (Ministério da Saúde). O consumo dos macronutrientes apresenta-se todos dentro da faixa de adequação, já que todos ficaram significativamente nos valores de referência, que são de 10 a $35 \%$ de proteína, 45 a $65 \%$ de carboidrato e 20 a $35 \%$ de lipídeo. ${ }^{26,27}$

Ao comparar as porções alimentares das mães com as porções alimentares da pirâmide alimentar, percebeu-se consumo adequado dos grupos de Arroz, Pães e Massas, Verduras e Legumes, Feijões e Oleaginosas, Carnes e Ovos e o grupo dos Açucares e Doces, já que não houve significância estatística entre a quantidade consumida e os valores de referência que são respectivamente 6, 3, e 1 para os três últimos grupos. Já o consumo dos grupos das Frutas, Leite, Queijo e Iogurtes, aparecem muito abaixo dos valores de referência. Houve grande diferença entre a quantidade consumida e o valor de referência, que é de 3 porções para ambos os grupos. O único grupo que apresentou consumo acima dos valores de referência foi o grupo dos Óleos e Gorduras, sendo seu valor de referência 1 porção no dia. ${ }^{28}$

Na pesquisa realizada por Dunker \& Philipi, ${ }^{12}$ foram avaliadas alunas de 15 a 18 anos de escolas públicas de São Paulo. Observou-se que aquelas com sintomas de AN possuíam aversão a determinados grupos de alimentos, como balas, chocolates em barra, refrigerante, macarrão 
e batata frita, alimentos considerados preferenciais entre o grupo sem sintomas de AN. Outro estudo semelhante realizado por Stracieri \& Oliveira, ${ }^{29}$ ao verificar o comportamento alimentar associado à ingestão de carboidratos, os autores perceberam que os alimentos ricos em carboidratos e açúcares são mais evitados pelas pacientes com AN, devido ao fato de essas pacientes acreditarem que este nutriente está associado ao ganho de peso. Comparando as pesquisas realizadas por Dunker \& Philipi ${ }^{12}$ e Stracieri \& Oliveira ${ }^{29}$ com o presente estudo, pode-se notar que as restrições alimentares presentes em pacientes com $\mathrm{AN}$, não se assemelham com o comportamento alimentar das mães aqui estudadas. ${ }^{24}$

Ao avaliar o EAT 26 notou-se valor próximo do risco para desenvolvimento de transtornos alimentares. Considerando que hoje em dia o EAT 26 é um dos métodos mais utilizado para auxiliar na triagem e diagnóstico de TA, valores positivos para este teste podem contribuir para o desenvolvimento e/ou manutenção do quadro de TA. ${ }^{28}$ Tal comportamento alimentar entre mães está cada vez maior, aumentando o risco do desenvolvimento de TA principalmente entre adolescentes e universitárias. ${ }^{30}$

Vários estudos são realizados com grupos de risco para o desenvolvimento de TA, dentre eles estão as bailarinas, atletas e estudantes de nutrição. Uma pesquisa com estudantes do curso de nutrição em um Centro Universitário de Ipatinga MG, mostrou que das 169 estudantes avaliadas, $50(29,59 \%)$ apresentaram resultado positivo no EAT, um dado muito preocupante, já que o TA não é uma patologia comum na sociedade. ${ }^{29}$ Em estudo com atletas do sexo feminino de diversas modalidades, não foram identificadas alterações na conduta alimentar, com resultado negativo para o EAT-26. Porém, um estudo com atletas do nado sincronizado e da ginastica rítmica, mostraram resultados positivos para o EAT-26. ${ }^{31}$

Paredes, Nessier \& González avaliaram dançarinas do Liceu Municipal da cidade de Santa Fé que tinham idades entre 13 e 23 anos, este estudo mostrou resultados positivos em 58,3\% para o EAT-40, teste que originou o EAT-26 usado no presente estudo. ${ }^{32}$

Ao analisar 413 adolescentes de ambos os sexos sendo 40 atletas de alto rendimento, 245 praticante de ginastica artística de categoria de base e 128 adolescentes não atletas, cujo objetivo era associar insatisfação corporal e influência da mídia, perfeccionismo, estado de humor e comportamento de risco para desenvolver TA, Neves et al. ${ }^{33}$ mostrou que 33,9\% dos atletas de base e 54,3\% dos atletas de alto rendimento apresentavam insatisfação corporal, explicado apenas pelo comportamento de risco para desenvolver TA, este fato foi associado a grande pressão externa para a busca e manutenção do corpo perfeito. ${ }^{33}$

Pike \& Rodin ${ }^{34}$ descobriram que as mães de adolescentes com TA eram mais propensas a ter TA do que as mães de adolescentes que não possuíam o TA. Recentemente, Elfhag \& Linné ${ }^{35}$ encontraram uma correlação significativa entre mães e filhas com TA. Um estudo realizado em Osona em 20 escolas com 3.147 alunos, junto com os pais identificou que $10 \%$ das meninas 
mostraram atitudes alimentares inadequadas, com pontuação para o EAT-26 acima de 20 pontos. Entre os pais, $3 \%$ dos pais e $8 \%$ das mães apresentaram este mesmo resultado positivo para o teste. ${ }^{36}$

De acordo com o estado nutricional das mães notou-se uma grande prevalência do sobrepeso e da Obesidade. Ao analisar a escala de figuras de silhuetas nota-se uma preocupação significativa com o peso em relação à imagem corporal, quando se observa as diferenças entre IMC real (aferido) e desejado. $\mathrm{O}$ uso de métodos de emagrecimento inadequados e a insatisfação com a imagem corporal podem ser fatores de risco para o desenvolvimento de TA. ${ }^{24}$ Porém, pesquisa realizada com 35 mães de pacientes adolescentes do sexo feminino (entre 10 e 17 anos) com TA, no Projeto Interdisciplinar de Atendimento, Ensino e Pesquisa em Transtornos Alimentares na Infância e Adolescência (PROTAD) do IPq - HC-FMUSP, concluiu que mães de pacientes adolescentes do sexo feminino com TA não apresentaram níveis de insatisfação corporal diferentes das encontradas em mães de filhas adolescentes sem TA, confirmando assim, a hipótese de que esta alteração em relação à imagem corporal, pode ser somente uma insatisfação presente na maioria das mulheres. ${ }^{37}$

É indiscutível a influência que a mãe pode ter no desenvolvimento do TA de sua filha, já que o primeiro vínculo criado pela criança é com a mãe no momento da amamentação. ${ }^{6}$ As influências hereditárias são a base da suscetibilidade tanto da AN quanto da BN. ${ }^{38}$

Como a relação mãe-filha em pacientes com TA é intensa, é necessário e de grande importância a participação da família no tratamento, o que corrobora o estudo realizado em 16 famílias que participaram de 16 sessões de tratamento em grupo. Observou-se que a participação familiar no tratamento foi essencial para o manejo do TA de seus filhos, contribuindo para o decréscimo do sofrimento com a doença pelos mesmos. ${ }^{39}$

Pode-se concluir que as mães estudadas apresentaram insatisfação com a imagem corporal, e nota-se grande prevalência de sobrepeso e obesidade no grupo estudado. Os hábitos e comportamentos alimentares não são semelhantes aos hábitos de pacientes portadores de TA, porém deve-se atentar a esse grupo, já que pode ser considerado de risco para desenvolvimento e/ou manutenção do quadro e de grande valia para o tratamento de suas filhas. Ressalta-se, assim, a importância da família no mesmo. Poucos estudos foram realizados avaliando o comportamento alimentar de mães de pacientes com transtornos alimentares, portanto é necessário realizar mais pesquisas envolvendo esse aspecto.

\section{Colaboradores}

Garcia CL e Miguel RM participaram na concepção e desenho do estudo, na coleta dos dados e na elaboração do artigo. Manochio-Pina MG e Pessa RP fizeram a revisão crítica de seu conteúdo e aprovação da versão final.

Conflito de Interesses: Os autores declaram não haver conflito de interesses. 


\section{Referências}

1. Reis JA, Silva Júnior CRR, Pinho L. Factors associated with the risk of eating disorders among academics in the area of health. Revista Gaúcha de Enfermagem. 2014; 35(2):73-78.

2. Gonçalves JA, Moreira EAM, Trindade EBSM, Fiates GMR. Transtornos alimentares na infância e na adolescência. Revista Paulista de Pediatria. 2013; 31(1):96-103.

3. Leal GVS, Philippi ST, Polacow VO, Cordás TA, Alvarenga MS. O que é comportamento de risco para transtornos alimentares em adolescentes? Jornal Brasileiro de Psiquiatria. 2013; 62(1):62-75.

4. Souza LV, Santos MA. Proximidade afetiva no relacionamento profissional-paciente no tratamento dos transtornos alimentares. Psicologia em Estudo. 2013; 18(3):395-404.

5. Polivy J, Herman CP. Causes of eating disorders. Annual Review of Psychology. 2002; 53(1):187-213.

6. Moura FEGA, Santos MA, Ribeiro RPP. A constituição da relação mãe-filha e o desenvolvimento dos transtornos alimentares. Estudos de Psicologia. 2015; 32(2):233-247.

7. Kaës R. Introdução ao conceito de transmissão psíquica no pensamento de Freud. In: Kaes R, organizador. Transmissão da vida psíquica entre as gerações. São Paulo: Casa do Psicólogo; 2001. p. 27-69.

8. Adami-Lauand C, Ribeiro R. A herança transgeracional nos transtornos alimentares: algumas reflexões. Psicologia USP. 2011; 22(4):927-942.

9. Pereira T, Lock J, Oggins J. Role of therapeutic alliance in family therapy for adolescent anorexia nervosa. Int J Eat Disord. 2006; 39(8):677-684.

10. Sopezki D, Vaz CE. O impacto da relação mãe-filha no desenvolvimento da autoestima e nos transtornos alimentares. Interação em Psicologia. 2008; 12(2):267-275.

11. Brasil. Ministério da Saúde. Vigilância Alimentar e Nutricional. Sisvan: orientações básicas para a coleta, processamento, análise de dados e informações em serviços de saúde. Brasília: Ministério da Saúde; 2004.

12. Dunker KLL, Philippi ST. Recordatório alimentar de 24 horas" modificado" avaliação do consumo alimentar de adolescentes. Anais do Congresso Latino-Americano de Nutrição Humana; 1999; Gramado.

13. Brasil. Ministério da Saúde. Guia alimentar: como ter uma alimentação saudável. Brasília: Ministério da Saúde; 2013.

14. Magalhães VC, Gas M. Transtornos alimentares em universitárias: estudo de confiabilidade da versão brasileira de questionários autopreenchíveis. Rev Bras Epidemiol. 2005; 8(3):236-245.

15. Garner DM, Garfinkel PE. The Eating Attitudes Test: an index of the symptoms of anorexia nervosa. Psychological Medicine. 1979; 9(2):273-279.

16. Bighetti F, Santos CB, Santos JE, Ribeiro RPP. Tradução e validação do eating attitudes test em adolescentes do sexo feminino de Ribeirão Preto, São Paulo. J Bras Psiquiatr. 2004; 6(53):339-346. 
17. Moraes C, Anjos LAD, Marinho SMSA. Construção, adaptação e validação de escalas de silhuetas para autoavaliação do estado nutricional: uma revisão sistemática da literatura. Cad Saúde Pública. 2012; 28:7-19.

18. Kakeshita IS, Silva AIP, Zanatta DP, Almeida SS. Construção e fidedignidade teste-reteste de escalas de silhuetas brasileiras para adultos e crianças. Psicologia: Teoria e Pesquisa. 2009; 25(2):263-270.

19. Siegel S, Castellan Junior NJ. Estatística não-paramétrica para ciências do comportamento. Porto Alegre: Artmed; 2006.

20. Silva GA, Costa KA, Giugliani ER. Infant feeding: beyond the nutritional aspects. Jornal de Pediatria. 2016; 92(3):S2-S7.

21. Bechara APV, Kohatsu LN. Tratamento nutricional da anorexia e da bulimia nervosas: aspectos psicológicos dos pacientes, de suas famílias e das nutricionistas. Vínculo. 2014; 11(2):07-18.

22. Neumark-Sztainer D, Bauer KW, Friend S, Hannan PJ, Story M, Berge JM. Family weight talk and dieting: how much do they matter for body dissatisfaction and disordered eating behaviors in adolescent girls? Journal of Adolescent Health. 2010; 47(3):270-276.

23. Linville D, Stice E, Gau J, O’Neil M. Predictive effects of mother and peer influences on increases in adolescent eating disorder risk factors and symptoms: a 3-year longitudinal study. Int J Eat Disord. 2011; 44(8):745-751.

24. Albino EBS, Macêdo EMC. Transtornos alimentares na adolescência: uma revisão de literatura. Veredas Fafip. 2014; 7:223-279.

25. Scagliusi FB, Pereira PR, Stelmo IC, Unsain RF, Martins PA, Sato PM. Insatisfação corporal, prática de dietas e comportamentos de risco para transtornos alimentares em mães residentes em Santos. Jornal Brasileiro de Psiquiatria. 2012; 61(3):159-167.

26. Padovani RM, Amaya-Farfán J, Colugnati FAB, Domene SMÁ. Dietary reference intakes: aplicabilidade das tabelas em estudos nutricionais. Revista de Nutrição. 2006; 19(6):741-760.

27. Philippi ST. Pirâmide dos alimentos: fundamentos básicos da nutrição. Barueri: Manole; 2014.

28. Bighetti, F. Tradução e validação do Eating Attitudes Test (EAT-26) em adolescentes do sexo feminino na cidade de Ribeirão Preto - SP [Dissertação]. [Ribeirão Preto]: Universidade de São Paulo; 2013.

29. Stracieri APM, Oliveira TC. Fatores de risco para o desenvolvimento de transtornos alimentares em universitárias. NUTRIR GERAIS - Revista Digital de Nutrição. 2008; 2(3):2-11. Disponível em: https://www.unilestemg.br/nutrirgerais/downloads/artigos/volume3/artigo_2_rng_fatores_ de_risco.pdf

30. Miranda MR. A complexidade da relação mãe-filha nos transtornos alimentares: um olhar da psicanálise. Revista Cadernos da Ceppan. 2009; 4:7-19.

31. Fortes LS, Almeida SS, Ferreira MEC. Imagem corporal e transtornos alimentares em atletas adolescentes: uma revisão. Psicologia em Estudo. 2013; 18(4), 667-677.

32. Paredes F, Nessier C, González M. Percepción de imagen corporal y conductas alimentarias de riesgo en bailarinas de danza clásica del Liceo Municipal de la ciudad de Santa Fe. Diaeta. 2011, 29(136):18-24. 
33. Neves CM, Meireles JFF, Carvalho PHB, Almeida SS, Ferreira MEC. Body dissatisfaction among artistic gymnastics adolescent athletes and non-athletes. Rev Bras Cineantropom Desempenho Hum. 2016; 18(1):82-92.

34. Pike KM, Rodin J. Mothers, daughters, and disordered eating. Journal of Abnormal Psychology 1991; 100(2): 198-204.

35. Elfhag K, Linné Y. Gender differences in associations of eating pathology between mothers and their adolescent offspring. Obesity Research 2005; 13(6): 1070-1076.

36. Yanez AA, Peix MA, Atserias N, Arnau A, Brug J. Association of eating attitudes between teenage girls and their parents. International Journal of Social Psychiatry. 2007, 53(6): 507-513.

37. Cobelo AIW. Insatisfação com a imagem corporal e sintomas de transtorno alimentar, em mães de adolescentes com transtornos alimentares [Dissertação]. [São Paulo]: Faculdade de Medicina da Universidade de São Paulo; 2008.

38. Grange DL, Lock J, Loeb K, Nicholls D. O papel da família nos Transtornos Alimentares. International Journal of Eating Disorders. 2010; 43,1: 1-5.

39. Jaeger MAS, Seminotti N, Falceto OG. O grupo multifamiliar como recurso no tratamento dos transtornos alimentares. Revista de Psiquiatria do Rio Grande do Sul. 2011; 33(1):20-27

Recebido: 21 de abril, 2018

Revisado: 28 de junho, 2018

Aceito: 30 de julho, 2018 
